\title{
Very Late Activation Antigens on Rheumatoid Synovial Fluid T Lymphocytes Association with Stages of T Cell Activation
}

\author{
Martin E. Hemler, David Glass, Jonathan S. Coblyn, and Jennie G. Jacobson \\ Dana Farber Cancer Institute, Harvard Medical School; and Department of Rheumatology and Immunology, \\ Brigham and Women's Hospital, Boston, Massachusetts 02115
}

\begin{abstract}
Lymphocytes from the synovial fluid of eight out of eight rheumatoid arthritis (RA) patients had elevated very late activation antigen-1 (VLA-1) expression (10-36\% positive cells), whereas peripheral blood lymphocytes (PBL) from RA patients and healthy controls had low VLA-1 expression ( $0-6 \%$ positive cells). During 1-2 wk of in vitro culture, VLA-1 increased on synovial fluid cells but remained low on PBL. In comparison, the interleukin 2 receptor (IL-2 R) was less prominent than VLA-1 on fresh synovial fluid cells, did not increase on cultured synovial fluid T cells, but did increase greatly on cultured PBL. The mitogen PHA reversed or prevented the appearance of VLA-1+, IL-2 $\mathbf{R}^{-}$synovial fluid cells during in vitro culture, thus giving IL-2 R+, VLA-1 ${ }^{-}$cells. These results emphasize that $(a)$ VLA$1+$ SF cells are different from resting cells or IL-2 $R+$ activated PBL T cells, and (b) VLA-1 on synovial fluid T cells may be incompatible with mitogen stimulation. In addition, the VLA-2 heterodimer $\left(165,000 / 130,000\right.$ relative molecular mass $\left.\left[M_{\mathrm{r}}\right]\right)$ was regulated opposite to the VLA-1 heterodimer $(130,000 / 210,000$ $M_{r}$ ) on synovial lymphocytes, and thus the VLA-1/VLA-2 ratio is another indicator of the stage of $\mathrm{T}$ cell activation.
\end{abstract}

\section{Introduction}

Several activation antigens known to appear in vitro have been measured in vivo on $T$ cells from patients with rheumatoid arthritis (RA) ${ }^{1}$ in order to further understand the role of activated $\mathrm{T}$ cells in disease. $\mathrm{T}$ cells bearing elevated levels of the class II (Ia) activation antigens have been found in peripheral blood (1, 2), synovial fluid, and synovium of patients with this disease (2, 3). Other activation markers, including OKT10 (4), T305 (5), and interleukin 2 receptor (IL-2 R) (6), have also been found on synovial $\mathrm{T}$ cells in RA. These results are consistent with the hypotheses that tissue injury in RA involves activated T cells.

Although synovial $\mathrm{T}$ cells from $\mathrm{RA}$ patients have been clearly shown to have an activated phenotype, in many respects they appeared to be quite different from in vitro activated normal $\mathbf{T}$

Address correspondence to Dr. Hemler, Dana Farber Cancer Institute, Boston, MA 02115.

Received for publication 14 March 1986.

1. Abbreviations used in this paper: IL-2 R, interleukin 2 receptor; MFI, median fluorescence intensity; $M_{\mathrm{r}}$, relative molecular mass; PHA, phytohemagglutinin; RA, rheumatoid arthritis; VLA-1, very late activation antigen 1.

J. Clin. Invest.

(c) The American Society for Clinical Investigation, Inc.

0021-9738/86/09/0696/07 $\$ 1.00$

Volume 78, September 1986, 696-702 cells. Normal $\mathrm{T}$ lymphoblasts obtained from in vitro mitogen stimulation were found to contain transferrin receptors (7), were blastoid, and had more IL-2 R than Ia (6). In contrast, synovial $T$ cells did not have detectable transferrin receptors (4), were largely nonblastoid, and had a greater elevation of Ia than IL-2 $\mathbf{R}$ (6).

Additional antigens associated with $\mathrm{T}$ cell activation have now been described. The very late activation antigen-1 (VLA1) appears slowly on activated $T$ cells in vitro and is not highly expressed until 2-3 wk after initial $\mathrm{T}$ cell stimulation, well after both proliferation and IL-2 R levels have diminished (8-10). On the other hand, conditions of increased $T$ cell stimulation and proliferation corresponded to decreased VLA-1 expression at the same time that a large number of other cell surface activation antigens (such as Ia, transferrin receptor, 4F2 antigen, and OKT10 antigen) were elevated (Hemler, M. E., and J. G. Jacobson, manuscript submitted for publication). Thus it has been suggested that the $210,000 / 130,000$ relative molecular mass $\left(M_{\mathrm{r}}\right)$ heterodimer VLA-1 may define a novel very late stage of activation that is phenotypically and functionally distinct from the earlier stages of activation (8-10), and that VLA-1 is not really an activation antigen, but perhaps is better called a postactivation antigen (Hemler, M. E., and J. G. Jacobson, manuscript submitted for publication).

The $210,000 / 130,000 M_{\mathrm{r}}$ VLA-1 heterodimer is part of a larger family of proteins that includes another long-term $\mathrm{T}$ cell protein complex called VLA-2 $\left(165,000 / 130,000 M_{\mathrm{r}}\right)$. Biochemical studies have shown that these structures are noncovalently linked heterodimers with unique $\alpha$ and common $\beta$ subunits (11). Conditions that cause changes in VLA-1 expression generally had an opposite effect on VLA-2 expression. Thus, T cell stimulation with antigen, mitogen, or in some instances IL2 resulted in a decreased VLA-1/VLA-2 ratio, whether by decreasing VLA-1, increasing VLA-2, or by doing both at once (Hemler, M. E., and J. G. Jacobson, manuscript submitted for publication).

The unusually late appearance of VLA-1 compared with other antigens appearing on activated $T$ cells suggested that this marker will be especially useful for analyzing the characteristics of $\mathrm{T}$ lymphocytes involved in the chronic inflammation of RA. Studies to determine the extent of VLA-1 (and VLA-2) expression on peripheral blood and synovial fluid lymphocytes from RA patients in the context of $T$ cell activation are reported herein.

\section{Methods}

Peripheral blood and synovial fluid samples. Blood and synovial fluid samples were obtained from RA patients undergoing long-term followup in the Department of Rheumatology and Immunology, Brigham and Women's Hospital, Boston, MA. All patients had IgM rheumatoid factor in serum and met the American Rheumatism Association's criteria of the diagnosis of RA (12). The synovial fluid samples were obtained as part of the routine clinical management. Peripheral blood samples were 
drawn during the same clinic visit in five of the patients studied. Additional blood samples were drawn from healthy volunteers.

Antibodies. The monoclonal antibody (MAb) TS2/7 (IgG1) recognizes the $210,000 / 130,000 M_{\mathrm{r}}$ VLA-1 complex $(9,11)$. The MAb A-1A5 (IgG2b) recognizes the $130,000 M_{\mathrm{r}}$ subunit common to both VLA-2 $\left(165,000 / 130,000 M_{\mathrm{r}}\right)$ and VLA-1 $(8,11)$. The expression of IL-2 R was analyzed using the MAb 49.9 (13) and T3 was analyzed using OKT3 from Ortho Pharmaceutical, Raritan, NJ. The negative control MAb P3 (IgG1) is the antibody secreted by the myeloma line P3-X63Ag8 (14).

$T$ cell samples, in vitro cell cultures, and mitogen stimulation. The synovial fluid was suspended in $10 \mathrm{vol}$ of phosphate-buffered saline (PBS), centrifuged, and the cell pellet was then enriched for mononuclear cells by Ficoll-Hypaque separation. Blood samples were also enriched for mononuclear cells by Ficoll-Hypaque separation. For in vitro culture, IL-2 (1-2 U/ml as defined by Gillis et al. [15]) was added to all cultures two times per week. IL-2 was prepared from culture supernatant resulting from 3-d phytohemagglutinin (PHA) stimulation of peripheral blood lymphocytes (PBL), and partially purified by ammonium sulfate fractionation (16). PHA (Difco Laboratories, Inc., Detroit, MI) diluted $1 / 1000$ was used to stimulate $T$ cell cultures where indicated.

Quantitation of cell surface antigens and cell cycle analysis. Viable cells $\left(1-5 \times 10^{5}\right)$ from Ficoll-Hypaque centrifugation were suspended in $50-\mu$ l aliquots of PBS containing $0.5 \%$ bovine serum albumin and $50 \%$ human serum to block nonspecific MAb binding. Then specific mouse MAb $(1 \mu \mathrm{g}$ in $50 \mu \mathrm{l})$ was added, cells were incubated $30 \mathrm{~min}$ at $0^{\circ} \mathrm{C}$, washed two times, and then incubated with saturating amounts of fluorescein-conjugated $\mathrm{F}\left(\mathrm{ab}^{\prime}\right)_{2}$ goat anti-mouse Ig. After further washing (three times), fluorescence was quantitated using either a fluorescenceactivated cell sorter analyzer (Becton-Dickinson \& Co., Mountain View, $\mathrm{CA}$ ) or an EPICS V flow cytometer (Coulter Electronics, Inc., Hialeah, FL). Volume gates were set to maximize lymphocyte counting (verified by OKT3 analysis) and to minimize interference from other cell types, such as monocytes or granulocytes. The channel numbers corresponding to median fluorescence intensity on a log scale were converted to linear numbers using a standard curve and the negative control fluorescence was then subtracted. A measure of $1.0 \mathrm{U}$ of this median fluorescence intensity (MFI) is approximately equivalent to 50,000 sites per cell. The percent positive cells was determined as $(\mathrm{SP}-\mathrm{C}) /(\mathrm{T}-\mathrm{C}) \times 100$ where SP equals specifically stained cells, $C$ equals negative control staining, and $T$ equals total cells counted. Values for SP and $C$ were taken as the number of cells to the right of the point midway down the $\mathrm{C}$ peak. Because the intensity of TS2/7 binding was sometimes rather low (e.g., Fig. 1), it is important to note that TS2/7 is the same subclass (IgG1) as the negative control MAb (P3) and both TS2/7 and P3 were from the same source (culture supernatant) and used at the same concentration.

For cell cycle analysis, cells were permeabilized by treatment with $70 \%$ ethanol $\left(30 \mathrm{~min}, 0^{\circ} \mathrm{C}\right)$, washed in PBS, and then stained with propidium iodide $(10 \mu \mathrm{g} / \mathrm{ml})$ added to $5 \times 10^{6}$ cells in $0.5 \mathrm{ml}$. Red DNA staining was quantitated using an EPICS $\mathrm{V}$ flow cytometer.

Iodination and immunoprecipitation of cell surface proteins. Cells were labeled with ${ }^{125}$ I using chloroglycoluril, and then $1 \%$ Nonidet-P40 cell lysates were used for immunoprecipitation as previously described $(8,9)$. Immune complexes were adsorbed onto Staphyloccus aureus Cowan I strain and analyzed by sodium dodecyl sulfate-polyacrylamide gels $(7 \%)$. The protein standards myosin $\left(200,000 M_{\mathrm{r}}\right)$, phosphorylase $\left(92,500 M_{\mathrm{r}}\right)$, and albumin $\left(69,000 M_{\mathrm{r}}\right)$ were used to estimate relative molecular mass.

\section{Results}

$V L A-1$ on $T$ cells from $R A$ patients. Synovial fluid lymphocyte preparations were studied using cell surface staining and flow cytometry. A representative flow cytometry profile (Fig. 1) shows that a substantial percentage of the synovial fluid $T$ cells (70-80\% T3 positive) were positive for VLA-1, and the range for eight samples was 10-36\% (Fig. 2). The IL-2 R was elevated

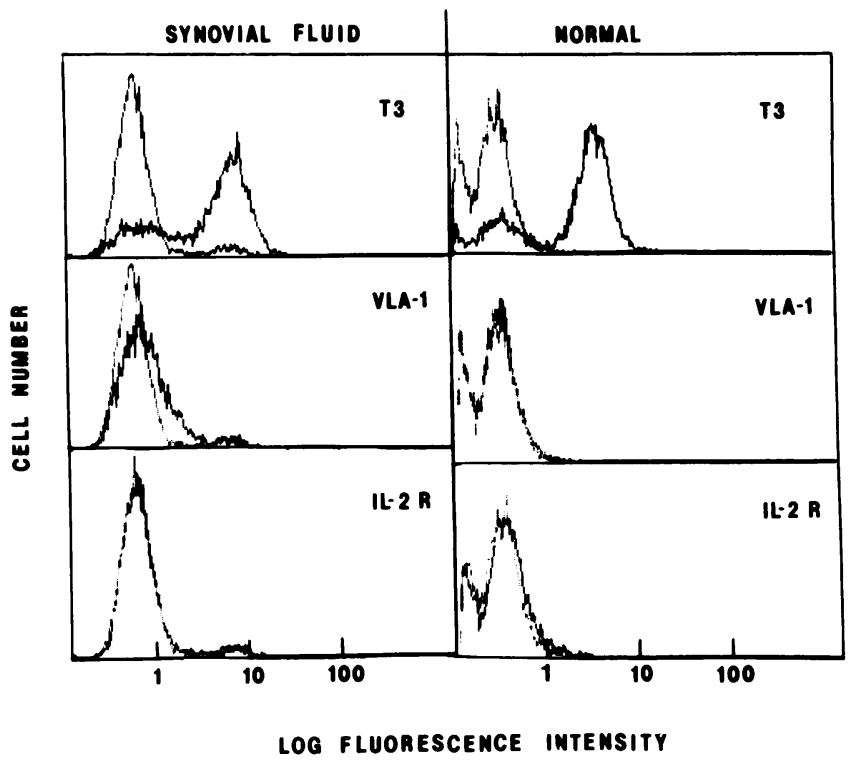

Figure 1. Comparison of VLA-1 and IL-2 R on T cells from synovial fluid and normal resting PBL T cells. Freshly isolated lymphocytes from the synovial fluid of an RA patient and the peripheral blood of a normal individual were analyzed by flow cytometry. Cells were stained with the Mab OKT3 (anti-T3), TS2/7 (anti-VLA-1), and 49.9 (antiIL-2 R).

in only two to three of the eight synovial fluid samples, and in all instances, fewer $T$ cells were positive for IL-2 $R$ than for VLA-1 (Figs. 1 and 2). In contrast, VLA-1 was minimally expressed (0-6\%) on nine samples of control PBL (Figs. 1 and 2) and on five samples of RA PBL (Fig. 2). The IL-2 R was found on $2-10 \%$ of these control and RA PBL, a reading a little higher than the readings for VLA-1. It is assumed that, when present, VLA-1 appears on T cells in these experiments because: $(a)$ the large majority of the synovial fluid cells that show rapid ampli-

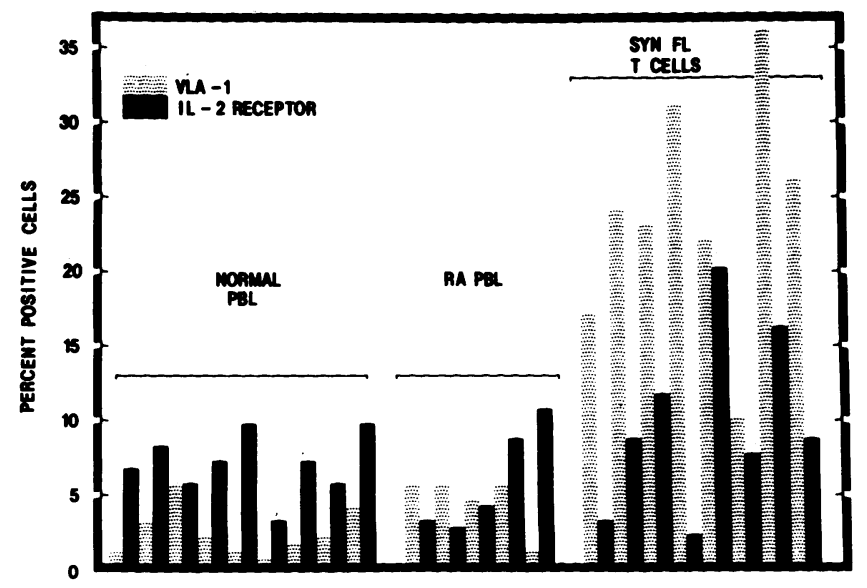

Figure 2. Comparison of VLA-1 and IL-2 R expression on a panel of freshly isolated $\mathrm{T}$ cell samples from RA patients and normal individuals. Lymphocyte (50-80\% T3 positive) from the peripheral blood of five RA patients and nine normals, and from the synovial fluid of eight RA patients were analyzed by flow cytometry. The percentage of cells positive for VLA-1 and IL-2 R was determined as described in Methods. Each pair of bars (dotted for VLA-1 and solid for IL-2 R) represents the data from one individual. 
fication of VLA-1 expression during in vitro culture are T3 positive cells (see below), (b) careful focusing of flow cytometer volume parameters on $\mathrm{T} 3$ positive cells caused an enrichment for VLA-1 positive cells, (c) cells expressing Leu 7, Leu 11, or OKM1 (natural killer cell markers) were not found in the populations analyzed, and $(d)$ there has been no evidence yet for VLA-1 appearance on either resting or activated B cells.

$V L A-1$ on cultured synovial fluid $T$ cells. The elevated level of VLA-1 expression that was seen in freshly isolated $T$ cells from RA synovial fluid (Figs. 1 and 2) was also seen when samples of those $T$ cells were cultured for $5 \mathrm{~d}$ in vitro in the presence of conditioned media (Fig. 3). In most cases the level of VLA1 expression was greatly amplified during culture (compare Figs. 3 and 1). Analyses of several synovial fluid samples during $3 \mathbf{w k}$ of in vitro culture showed the increase in VLA-1 expression was a consistent finding, but this elevation of VLA-1 was not necessarily maintained in culture, since it was followed by a marked decline during some experiments (Fig. 4).

In contrast, IL-2 $R$ expression on these cultured RA synovial fluid lymphocytes was markedly lower than VLA-1 expression (Fig. 3, left panels), and was not appreciably elevated on any of the samples tested (Fig. 4). These cells were not rapidly dividing (data not shown), which is consistent with their low IL-2 R expression. However, when control PBL T cells were cultured for $5 \mathrm{~d}$, the opposite result was obtained. Such cultures readily increased in cell numbers, had elevated IL-2 receptor, and had low expression of VLA-1 (Fig. 3, right panels). In the additional examples shown (Fig. 4), control and RA PBL cultures both had elevated IL-2 $R$ and low VLA-1 levels.

The effect of PHA stimulation on synovial fluid T cell VLA1 expression. The expression of elevated VLA-1 but not IL-2 R on synovial fluid $T$ cells (Figs. 1-4) suggested that perhaps these cells were not recently activated but were in a late-activated stage, characterized by expression of the very late activation antigen, VLA-1. Thus the mitogen PHA was used in a study to test the effects of reactivation on VLA-1 expression. When PHA was added at the beginning of an 11-d in vitro culture of synovial fluid T cells, the eventual appearance of VLA-1 was greatly su-

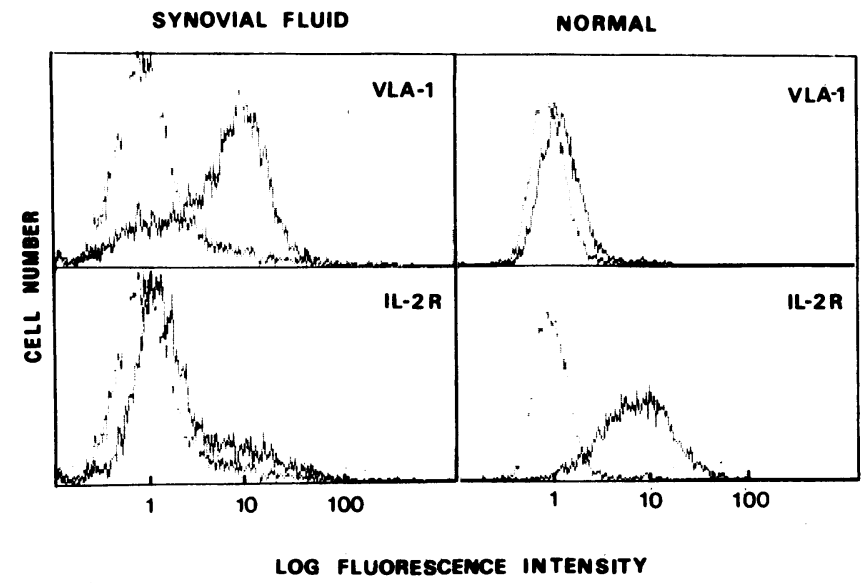

Figure 3. Comparison of VLA-1 and IL-2 R expression on in vitro cultured synovial fluid $T$ cells and normal $T$ cells. $T$ cells from synovial fluid and normal PBL were cultured in the presence of partially purified IL-2 (1-2 U/ml) without any other stimulation. After $5 \mathrm{~d}$, cells were analyzed for VLA-1 and IL-2 R expression by flow cytometry.

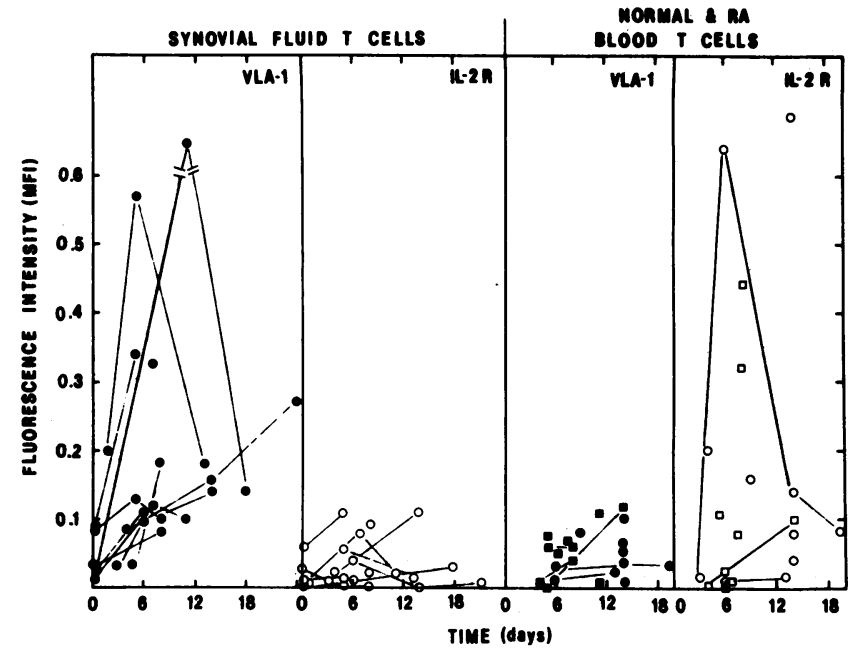

Figure 4. Expression of VLA-1 and IL-2 R on a panel of in vitro cultured T cell samples. After in vitro culture (as in Fig. 3 ) for the indicated times ( $y$ axis), synovial fluid T cells (left panels), and peripheral blood T cells (right panels) were analyzed for VLA-1 and IL-2 R expression. Analyses of PBL (right panels) include PBL from both normals $(\bullet, O)$ and RA patients $(\square, \square)$. Immunofluorescence is quantitated as the MFI that is proportional to the number of molecules per cell. In this figure it was more informative to graph the MFI values rather than the percentage of positive cells, because there was a wide range of MFI values on populations that were nearly $100 \%$ positive. Connected points represent samples analyzed at different times from the same individual culture.

pressed compared with an identical culture without PHA (Fig. 5). In another experiment, PHA was added after synovial fluid $\mathrm{T}$ cells had already been cultured for $24 \mathrm{~d}$ in vitro (Fig. 6). In that instance, VLA-1 (which had been elevated) disappeared by day 33, whereas IL-2 $R$ (which was absent) became prominent by day 33. In experiments such as the one shown in Fig. 6, time points were not obtained sooner after PHA addition because excessive cell clumping prevented accurate assessment of cell surface antigen levels for several days immediately after PHA addition. The cell surface expression of the T3 antigen was not

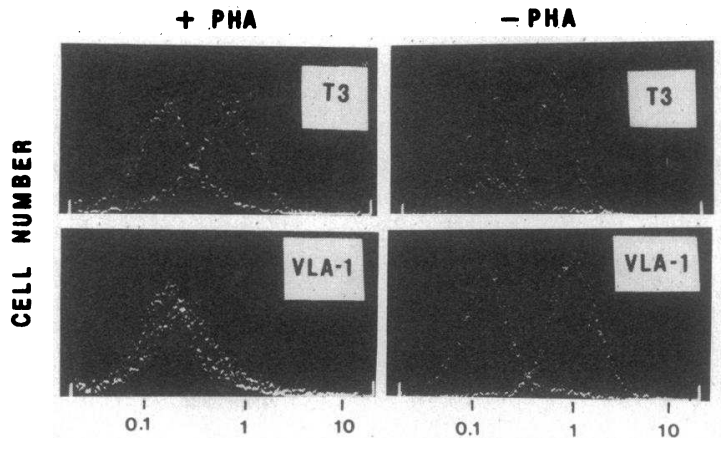

LOG FLUORESCENCE INTENSITY

Figure 5. The effect of PHA on the initial expression of VLA-1 on synovial fluid $T$ cells during in vitro culture. Synovial fluid $T$ cells were cultured in the presence (left panels) or absence (right panels) of PHA for $5 \mathrm{~d}$. Then the expression of the cell surface antigens T3 and VLA-1 was analyzed by flow cytometry. 
A. (+) PHA RESTIM.

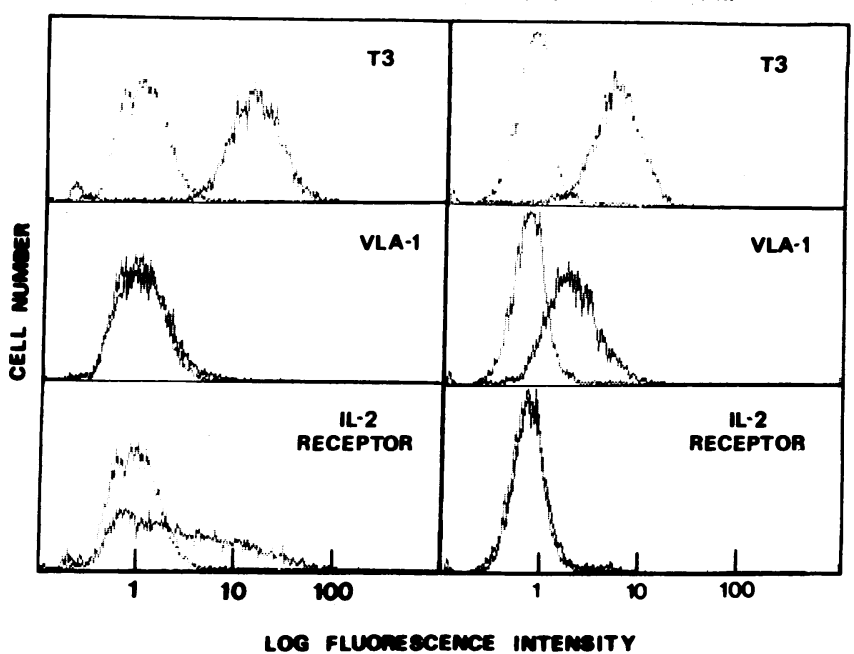

Figure 6. The effect of PHA on cultured synovial T cells already expressing substantial VLA-1 levels. Synovial fluid T cells were initially stimulated with PHA and then cultured for $33 \mathrm{~d}$ without further PHA stimulation (right panels). Alternatively, PHA was added again at day $24,9 \mathrm{~d}$ before assay (left panels). After $33 \mathrm{~d}$, each culture was analyzed by flow cytometry for T3, VLA-1, and IL-2 R.

notably affected in either experiment (Figs. 5 and 6). In further experiments, the expression of VLA-1 on six out of six in vitro cultures of synovial fluid cells was suppressed when PHA was included, and the mitogen concanavalin A had a similar effect on VLA-1 expression (data not shown). In each case (e.g., as seen in Fig. 6) the level of IL-2 R expression was markedly elevated after mitogen stimulation (data not shown).

At the same time that VLA-1 and IL-2 R analyses were carried out (Fig. 6), cells were also analyzed for cell cycle distribution using propidium iodide. The results on day 33 indicated that $32 \%$ of the PHA-restimulated cells were in the proliferative phases of the cell cycle $\left(S, G_{2}, M\right)$ whereas the nonrestimulated cells had only $9 \%$ in those cell cycle phases. These results confirm that PHA had a mitogenic effect on cultured synovial $\mathrm{T}$ cells, and that VLA-1 was present on the less proliferative cells.

Cells cultured for $11 \mathrm{~d}$ in the absence of PHA (Fig. 5) appeared to be $\sim 30 \%$ negative for T3. At the same time, those cells appeared to be $\sim 100 \%$ positive for VLA-1. These results suggest that although VLA-1 expression has only been described on activated $T$ cells in vitro $(2,3)$, in at least some cases VLA1 expression is not limited to T3 positive lymphoid cells.

Immunoprecipitation of VLA-1 from synovial fluid $T$ cells. Immunoprecipitation of VLA-1 on synovial fluid T cells was carried out to verify independently the cell surface binding results (Figs. 1-6), and also to allow simultaneous comparisons of VLA1 and VLA-2 expression. The Mab A-1A5 was used in these studies because it binds to the common $\beta$ subunit of both VLA1 and VLA-2 and thus immunoprecipitates both structures at once. The $\alpha^{1}$ protein of VLA-1 $\left(210,000 / 130,000 M_{\mathrm{r}}\right)$ along with the detectable $\alpha^{2}$ protein of VLA-2 $\left(160,000 / 130,000 M_{\mathrm{r}}\right)$ was readily immunoprecipitated from freshly isolated synovial fluid cells (Fig. $7 A$, lane $a$ ). Immunoprecipitations of other synovial fluid samples gave similar results (data not shown). In contrast to the result in Fig. 7 (lane $a$ ), immunoprecipitation from normal PBL (lane $b$ ) showed that the $\alpha^{1}$ portion of VLA-1 was barely evident relative to the intensity of the $\beta$ subunit. This result is consistent with the flow cytometry results (Figs. 1 and 2) showing elevated VLA-1 on synovial fluid cells compared with PBL. This PBL result also agrees with previous results $(8,9)$, in which the $\beta$ subunit was immunoprecipitated from PBL in the absence of detectable $\alpha$ subunits. When cultured in the absence of PHA restimulation, synovial fluid T cells showed high $\alpha^{1}$ protein relative to moderate $\beta$ and very weak $\alpha^{2}$ expression (Fig. $7 \mathrm{~B}$, lane c). In contrast, after PHA restimulation, $\alpha^{1}$ was weak relative to $\beta$ and slightly less intense than $\alpha^{2}$ (lane $e$ ). Thus, PHA treatment caused a marked decrease in the VLA-1/VLA-2 ratio on synovial fluid $\mathrm{T}$ cells. The experiments shown in lanes $c$ and $e$ carried out using the MAb A-1A5 were repeated using the MAb TS2/ 7, which only recognizes VLA-1 (lanes $d$ and $f$ ). These results again showed that VLA-1 was elevated in the absence of PHA restimulation (lane $d$ ) and diminished after PHA restimulation (lane $f$ ) in agreement with the cell surface binding results (Figs. 5 and 6). Since a decrease in VLA-1 was observed (Fig. 7 B) using two different MAb, which recognize different subunits of the heterodimer, it is unlikely that PHA binding directly to the VLA-1 complex was interfering with VLA-1 detection.

A comparison of synovial fluid cells and control PBL, each cultured for $7 \mathrm{~d}$ in vitro, was also carried out (Fig. $7 \mathrm{C}$ ). Immunoprecipitation with the MAb A-1A5 showed that the $\alpha^{1}$ protein of VLA-1 was present in nearly equal intensity as $\beta$ on synovial $\mathrm{T}$ cells in culture (lane $h$ ), whereas cultured control PBL had only a trace of detectable $\alpha^{1}$ protein relative to the prominent $\beta$ protein (lane $j$ ). Thus, the immunoprecipitation result is consistent with VLA-1 being low or absent on cultured control cells compared with cultured synovial fluid cells (See Figs. 3 and 4, above). It is also evident that $\alpha^{1}$ is present well in

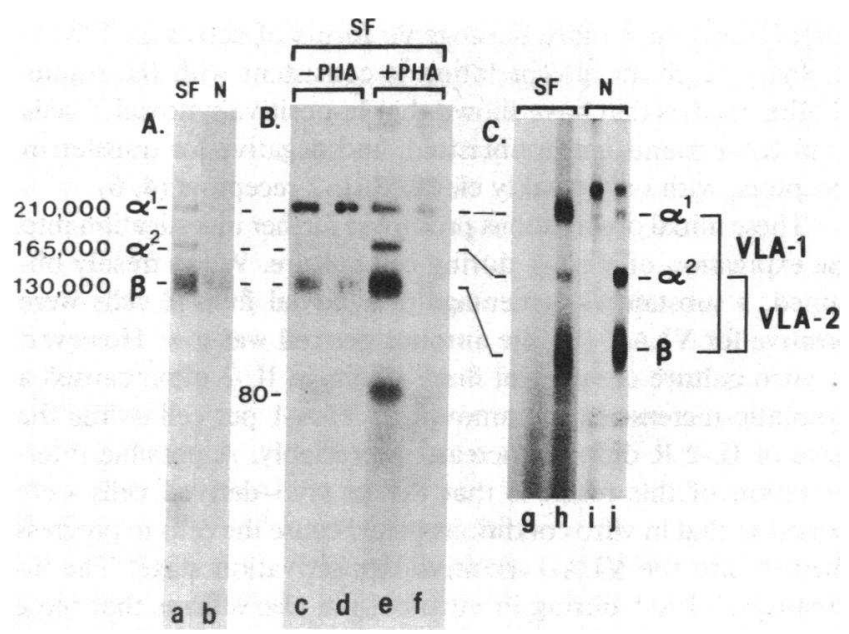

Figure 7. Immunoprecipitation of VLA proteins from fresh and cultured synovial fluid T cells. $(A)$ Freshly isolated synovial fluid lymphocytes (SF, lane $a$ ) and normal PBL (N, lane $b$ ) were ${ }^{125}$ I-radiolabeled and immunoprecipitated using the Mab A-1A5 as described in Methods. (B) Synovial fluid T cells were initially stimulated with PHA, then cultured for $33 \mathrm{~d}$ in the presence of partially purified IL-2 with (lanes $c$ and $d$ ) or without (lanes $e$ and $f$ ) the further addition of PHA on day 24. Immunoprecipitations were with the MAb A-1A5 (lanes $c$ and $e$ ) and TS2/7 (lanes $d$ and $f$ ). ( $C$ ) Synovial fluid T cells (lanes $g$ and $h$ ) and normal PBL (lanes $i$ and $j$ ) were cultured for $7 \mathrm{~d}$ and then analyzed using the MAb A-1A5 (lanes $h$ and $j$ ) or the negative control P3 (lanes $g$ and $i$ ). 
excess of $\alpha^{2}$ protein in lane $h$, whereas $\alpha^{1}$ is hardly detectable relative to $\alpha^{2}$ in lane $j$. Thus, the ratio of VLA-1/VLA-2 is vastly greater for the cultured synovial cells compared with the cultured normal cells. Control immunoprecipitations (lanes $g$ and $i$ ) show nonspecific background bands. The band that appears just above $\alpha^{1}$ (lane $j$ ) also appears in the control lane (lane $i$ ) and thus should not be confused with $\alpha^{1}$. Also, a prominent $80,000-M_{\mathrm{r}}$ protein is seen in lane $e$. A similar protein has been variably observed in previous VLA immunoprecipitations $(8,9,12)$, but its exact relationship to VLA proteins has not yet been defined.

\section{Discussion}

The results in this paper demonstrate that VLA-1, an antigen associated with late stages of $T$ cell activation, is expressed on synovial fluid cells from patients with RA. In contrast to the joint findings, VLA-1 was not found on PBL from either RA patients or from controls. The observation of VLA-1 positive T cells in the synovium, but not in the peripheral blood of RA patients, supports the concept that synovial $\mathrm{T}$ cells represent a distinct compartmentalized population separate from PBL. This is in agreement with other studies that have shown major phenotypic and functional differences between those $T$ cell populations $(3,17,18)$.

Earlier studies in vitro have shown that VLA-1 was rarely present on mitogen-stimulated $\mathrm{T}$ cell blasts until after the levels of blast-associated structures, such as IL-2 R and transferrin receptor, had diminished. Thus it seems likely that VLA-1 defines a new stage of late activation on T cells $(10,19)$. In this context it is noteworthy that the IL-2 $R$ was present on synovial fluid lymphocytes to a much lesser degree than VLA-1. This suggests that the synovial fluid lymphocyte in RA is activated late and differs from other more blastogenic forms of activated $T$ lymphocytes. Such an interpretation is consistent with the results of other studies that have shown that la-positive synovial $T$ cells from RA patients are nonblastoid, and negative for transferrin receptors, with only weakly elevated IL-2 receptors $(4,6)$.

These initial observations prompted further investigation into the expression of VLA-1 during cell culture. When freshly obtained, a substantial percentage of synovial fluid $T$ cells were positive for VLA-1 but the amount per cell was low. However, in vitro culture of synovial fluid T cells in IL-2 often caused a dramatic increase in the amount of VLA-1 per cell, while the level of IL-2 R did not increase appreciably. A possible interpretation of this result is that the in vivo-derived cells were poised so that in vitro conditions would cause the cells to progress further into the VLA-1-positive late-activation stage. The increase in VLA-1 during in vitro culture also affirms that these cells are fully capable of synthesizing VLA-1 rather than acquiring it from some other cell source. Even though the expression of IL-2 R remained low on cultured synovial $T$ cells these cells still required IL-2 for maintenance of in vitro viability, since removal of IL-2 caused cell death. Nonetheless, the continued lack of expression of substantial levels of IL-2 R suggests that these cells are not being reactivated in culture. This finding appears to be consistent with previous reports that have indicated that synovial $T$ cells are relatively insensitive to in vitro activation, whether by mitogens $(20)$ or by autologous stimulator cells (17). Interestingly, in some in vitro culture experiments, the high levels of VLA-1 expression were transient, suggesting additional in vitro regulation (down as well as up) of VLA-1 expression. At present the precise conditions or factors regulating these changes in VLA-1 are not understood.

In contrast to synovial fluid $T$ cells, PBL did not express markedly elevated VLA-1 during the first few weeks of in vitro culture. This is in agreement with previous results showing delayed VLA-1 expression on in vitro cultured T cells (8-10). Also in contrast to synovial fluid T cells, variably elevated IL-2 receptor expression was seen, indicating that in vitro activation was occurring. The exact nature of this activation was not analyzed, but it probably represents some form of lymphokineactivated killer cell selection (21) or stimulation by serum components or autologous cells. These results indicating major differences between in vitro cultures of synovial $T$ cells and PBL provide further evidence that these two cell populations represent very different activated cell stages or cell types.

When mitogen was added either at the beginning or after in vitro culture of synovial T cells in the presence of IL-2, it appeared that $\mathrm{T}$ cell recycling occurred with concomitant loss of VLA-1 expression and gain of IL-2 $R$ expression. As these late stage-activated $T$ cells can be restimulated, they are not irreversibly committed to the VLA-1-positive, low IL-2 R stage. As an alternative to the recycling concept, it is possible that mitogens could selectively stimulate a small subpopulation of VLA-1negative cultured synovial $T$ cells that would then expand and outgrow the VLA-1-positive cells in culture. The latter explanation appears less likely, since there has been no evidence of intermediate subpopulations that would have resulted in multiple VLA-1 peaks detected by flow cytometry.

Limited cell cycle analysis in this paper confirmed that VLA1 is present on less proliferative cells. However, while VLA-1 expression is favored on more quiescent cells, VLA-1 cannot simply be regarded as a marker for cell quiescence. There are counterexamples, such as $T$ cell cultures that have been maintained in vitro for several months and did not lose VLA-1 when stimulated (Hemler, M. E., and J. G. Jacobson, manuscript submitted for publication) and, of course, PBL are quiescent but do not express VLA-1. Also, simultaneous analysis of cell cycle and VLA-1 expression did not show a difference in VLA-1 levels on cells in $S, G_{2}$, or $M$ compared with $G_{1} / G_{0}$ (data not shown). Rather, it appears that VLA-1 slowly accumulates on cells, particularly when they remain quiescent for extended periods. Further experiments are needed to characterize more precisely any possible relationship between VLA-1 and cell cycle.

Although results from these and other studies show that IL$2 \mathrm{R}$ and VLA-1 expression can be inversely regulated by $T$ cell growth conditions, it would be incorrect to imply that there is no overlap in the expression of those two structures. For example, human T cell leukemia/lymphotrophic virus 1-infected cultures (9) and some long-term T cell lines and clones (10) can sometimes simultaneously express relatively high levels of both structures. Also, results with $\mathrm{T}$ cell clones have indicated that both IL-2 R and VLA-1 can be simultaneously expressed, although the relative amounts vary widely as they are regulated differently by cell stimulation (Hemler, M. E., and J. G. Jacobson, manuscript submitted for publication).

Besides analyzing VLA-1 and IL-2 R on activated T cells, we also compared VLA-1/VLA-2 ratios. Although a MAb that specifically recognizes VLA-2 has not yet been described, this $165,000 / 130,000-M_{\mathrm{r}}$ heterodimer can readily be seen with VLA1 by immunoprecipitation using the MAb A-1A5 $(8,11)$. As the 
immunoprecipitation data in this paper showed, the VLA-1/ VLA-2 ratio was substantially diminished on in vitro cultures of normal compared with synovial fluid cells and again on PHAstimulated compared with unstimulated cultures of synovial fluid cells. In both cases, the difference in ratio was not only due to lower VLA-1, but also to elevated VLA-2. Thus, analysis of this ratio may be even more indicative of growth differences between activated $T$ cell populations than analysis of VLA-1 alone. In these experiments, it was clear that PHA-stimulated RA synovial fluid cells are more like normal cultured cells with respect to their VLA-1/VLA-2 ratio than they are when first drawn from an affected joint. This provides additional evidence that suggests that synovial $T$ cells can be recycled out of their very late activated stage.

Although in this initial study of VLA-1 on RA T cells, there has been no attempt to make detailed clinical correlations with levels of VLA-1 expression, data is already available to suggest that these findings are not unique to RA. Lymphocytes from patients with multiple sclerosis have been analyzed for VLA-1 expression (22), and a significant percentage had elevated VLA1 on their PBL T cells, but little or no elevation in the levels of early activation antigens (IL-2 R and 4F2). Activated $T$ cells also occur in normal lung, and such $T$ cells are known to be Iapositive, but nonproliferating and negative for transferrin and IL-2 receptors (23). Considering those similarities to RA synovial $T$ cells, it is not surprising that lung $T$ cells have recently been shown to be significantly elevated for VLA-1 expression (24).

In the future the inclusion of VLA-1 with analyses of the other activation-related structures, which define different types of stages of activated T cells, should expand our ability to interpret the role of in vivo activated $T$ cells in a variety of normal and disease situations.

In summary, the results in this paper illustrate that $(a)$ elevated VLA-1 expression can occur on synovial fluid $T$ cells in vivo and expression is amplified during in vitro culture, $(b)$ in contrast to normal T cells, VLA-1 expression is higher than IL2 receptor expression on synovial fluid $\mathrm{T}$ cells both in vivo and after in vitro culture, $(c)$ PHA stimulation can reverse or prevent the appearance of VLA-1-positive $\mathrm{T}$ cells in in vitro synovial fluid cell cultures, and $(d)$ the VLA-1/VLA-2 ratio may even be a stronger indicator of relative states or stages of $\mathrm{T}$ cell activation than VLA-1 expression alone. Thus, VLA-1 together with VLA2 and the IL-2 $R$ help to define differences in stages of $T$ cell activation relevant to the pathogenesis of rheumatoid arthritis.

\section{Acknowledgments}

We thank Dr. M. Brenner and Dr. J. L. Strominger for advice and support, and Christina Huang for assistance with immunoprecipitations.

This work was supported by grant IM-393 from the American Cancer Society, by grants 2-PO-AI-21163, AM-30486, and AM-20580 from the National Institutes of Health, and by an Arthritis Foundation Investigator Award to Dr. Hemler.

\section{References}

1. Yu, D. T. Y., R. J. Winchester, S. M. Fu, A. Gibofsky, H. S. Ko, and H. G. Kunkel. 1980. Peripheral blood Ia-positive T cells. J. Exp. Med. 151:91-100.

2. Klareskog, L., U. Forsum, U. Malmnäs Tjernlund, D. Kabelitz, and A. Wigren. 1981. Appearance of anti-HLA-DR-reactive cells in normal and rheumatoid synovial tissue. Scand. J. Immunol. 14:183-192.

3. Burmester, G. R., D. T. Y. Yu, A. Irani, H. G. Kunkel, and R. J. Winchester. 1981. Ia + T cells in synovial fluid and tissues of patients with rheumatoid arthritis. Arthritis Rheum. 24:1370-1376.

4. Fox, R. I., S. Fong, N. Sabharwal, S. A. Carstens, P. C. Kung, and J. H. Vaughan. 1982. Synovial fluid lymphocytes differ from peripheral blood lymphocytes in patients with rheumatoid arthritis. J. Immunol. 128:351-354.

5. Fox, R. I., M. Hueniken, S. Fong, S. Behar, I. Royston, S. K. Singhal, and L. Thompson. 1983. A novel cell surface antigen (T305) found in increased frequency on acute leukemia cells and in autoimmune disease states. J. Immunol. 131:762-767.

6. Burmester, G. R., B. Jahn, M. Gramatzki, J. Zacher, and J. R. Kalden. 1984. Activated T cells in vivo and in vitro: divergence in expression of Tac and Ia antigens in the nonblastoid small $\mathrm{T}$ cells of inflammation and normal T cells activated in vitro. J. Immunol. 133:12301234.

7. Cotner, T., J. M. Williams, L. Christenson, H. M. Shapiro, T. B. Strom, and J. L. Strominger. 1983. Simultaneous flow cytometric analysis of human T cell activation antigen expression and DNA content. J. Exp. Med. 157:461-472.

8. Hemler, M. E., C. F. Ware, and J. L. Strominger. 1983. Characterization of a novel differentiation antigen complex recognized by a monoclonal antibody (A-1A5): unique activation-specific molecular forms on stimulated T cells. J. Immunol. 131:334-340.

9. Hemler, M. E., F. Sanchez-Madrid, T. J. Flotte, A. M. Krensky, S. J. Burakoff, A. K. Bhan, T. J. Springer, and J. L. Strominger. 1984. Glycoproteins of 210,000 and $130,000 \mathrm{M}$.W. on activated T cells: cell distribution and antigenic relation to components on resting cells and T cell lines. J. Immunol. 132:3011-3018.

10. Hemler, M. E., J. G. Jacobson, M. B. Brenner, and J. L. Strominger. 1985. VLA-1: a T cell surface antigen which defines a novel late stage of T cell activation. Eur. J. Immunol. 15:502-508.

11. Hemler, M. E., J. G. Jacobson, and J. L. Strominger. 1985. Biochemical characterization of VLA-1 and VLA-2: cell surface heterodimers on activated T cells. J. Biol. Chem. 260:15246-15252.

12. Ropes, M. W., E. A. Bennett, S. Cobb, R. Jacox, and R. Jessar. 1958. 1958 Revision of diagnostic criteria for rheumatic arthritis. Bull. Rheum. Dis. 9:175-181.

13. Hemler, M. E., B. Malissen, N. Rebai, A. Liabeuf, C. Mawas, K. M. Kourilsky, and J. L. Strominger. 1983. A 55,000 $\mathrm{M}_{\mathrm{r}}$ surface antigen on activated human $\mathrm{T}$ lymphocytes defined by a monoclonal antibody. Hum. Immunol. 8:153-165.

14. Kearney, J. F., A. Radbruch, B. Liesegang, and K. Rajewsky. 1979. A new mouse myeloma cell line that has lost immunoglobulin expression but permits the construction of antibody-secreting hybrid cell lines. J. Immunol. 123:1548-1550.

15. Gillis, S., M. Ferm, W. Ou, and K. A. Smith. 1978. T-cell growth factor: parameters of production and a quantitative microassay for activity. J. Immunol. 120:2027-2032.

16. Mier, J. W., and R. C. Gallo. 1980. Purification and some characteristics of human T-cell growth factor from phytohemagglutininstimulated lymphocyte-conditioned media. Proc. Natl. Acad. Sci. USA. 77:6134-6138.

17. Silver, R. M., D. Redelman, and N. J. Zvaifler. 1983. Studies of rheumatoid synovial fluid lymphocytes. II. A comparison of their behavior with blood mononuclear cells in the autologous mixed lymphocyte reaction and response to TCGF. Clin. Immunol. Immunopathol. 27:1527.

18. Kluin-Nelemans, H. C., J. A. van der Linden, F. H. J. Gmelig Meyling, and H.-J. Schuurman. 1984. HLA-DR positive T lymphocytes in blood and synovial fluid in rheumatoid arthritis. J. Rheumatol. 11: 272-276.

19. Hemler, M. E., J. Jacobsen, and J. L. Strominger. 1985. T cell stimulation by mitogen: inverse regulation of the expression of IL-2 re- 
ceptor and VLA-1 protein complexes on activated T cells. In Advances in Gene Technology: Molecular Biology of the Immune System. Proc. of the 17th Miami Winter Symposium. 2:177-178.

20. Ivanyi, L., T. Lehner, and H. C. Burry. 1973. The response of synovial fluid lymphocytes to $\mathrm{T}$ and $\mathrm{B}$ stimulants in vitro. Immunology. 25:905-908.

21. Grimm, E. A., R. J. Robb, J. A. Roth, L. M. Neckers, L. B. Lachman, D. J. Wilson, and S. A. Rosenberg. 1983. Lymphokine-activated killer cell phenomenon. III. Evidence that IL-2 is sufficient for direct activation of peripheral blood lymphocytes into lymphokine-activated killer cells. J. Exp. Med. 158:1356-1361.

22. Hafler, D. A., M. E. Hemler, L. Christenson, J. M. Williams,
H. M. Shapiro, T. B. Strom, J. L. Strominger, and H. L. Weiner. 1985. Investigation of in vivo $\mathrm{T}$ cells in multiple sclerosis and inflammatory central nervous system diseases. Clin. Immunol. Immunopathol. 37:163171.

23. Davidson, B. L., J. Faust, S. Pessano, R. P. Daniele, and G. Rovera. 1985. Differentiation and activation phenotypes of lung $T$ lymphocytes differ from those of circulating $\mathrm{T}$ lymphocytes. J. Clin. Invest. 76:60-65.

24. Saltini, C., M. Hemler, J. Spurzem, and R. Crystal. 1986. Expression of the "very late activation antigen" (VLA-1) complex on lung $T$-cells characterizes a population of activated nonproliferating T-cells compartmentalized in the normal lung. American Thoracic Society Abstracts. Am. Rev. Respir. Dis. 133:A242. (Abstr.) 